\title{
ORIGINAL
}

\section{SELECCIÓN DE INDICADORES PARA EVALUAR LA ADHESIÓN A UNA GUÍA FARMACOTERAPÉUTICA POR LOS MÉDICOS GENERALES DE UN ÁREA DE ATENCIÓN PRIMARIA}

\author{
Ana Isabel Rigueira García (1) y Vicente Gómez Juanes (2) \\ (1) Insuluud. Gerencia de Atención Primaria del Área Sanitaria III. - Avilés. Asturias. \\ (2) Subdirección General de Atención Primaria. Insalud.
}

\section{RESUMEN}

Fundamento: La medida continuada de la calidad de la prescripción farmacéutica forma parte de los programas de mejora de calidad de procesos asistenciales sanitarios en Atención Primaria, pero, en general, se carece de indicadores validados que puediun aúlizarse para realizar dicho proceso de evaluación. La medida de la adhesión a una selección de fármacos determinada, como una guía de área consensuada, puede utilizarse como indicador global de la calidad de prescripción. El objetivo del trabajo ha sido el establecimiento de un método para seleccionar indicadores de calidad de tipo globalizador, ejemplificando el mismo en selección de un indicador para medir la adhesión a la guía de áreal.

Métodos: Estudio descriptivo transversal utilizando como unidad de análisis los médicos generales del Área Sanitaria de Avilés $(n=78)$ y como fuente de información las recetas facturadas de los mismos durante 1997. Se elaboraron 7 indicidores para medir la adhesión a la Guía Farmacoterapéutica del Área. Se realizó una selección estudiando las siguientes características: fiabilidad, facilidad de obtención, precisión, sensibilidad, especificidad y coherencia.

Resultados: Las puntuaciones obtenidas oscilaron entre 10 y 23 (sobre un máximo posible de 28), siendo el valor más alto el correspondiente al porcentaje de recetas de principios activos de la guía con respecto al total de recetas prescritas por cadia facultativo.

Conclusiones: Los indicadores utilizados en la evaluación de la calidad farmacológica deben analizarse de un modo detallado previamente a su implantación. Se propone un método innovador para realizar la validación de indicadores de monitorización de la prescripción farmacéutica de tipo globalizador. De los indicadores estudiados para la medida de la a ahesión a la guía farmacéutica de Atención Primaria el más útil es el porcentaje de recetas de los principios activos de la misma respecto al total de recetas prescritas. Lia adhesión a la Guía Furm:ıcológica del Áreal se relaciona positivamente con una prescripción de calidad.

Palabras clave: Guía Farmacoterapéutica. Formulario Atención primaria. Prescripción. Uso de medicamentos. Control de calidad. Indicadores de calidad. Evaluación. Sistemas de información.

Correspondencia:

Ana Isabel Rigueira García

Gerencia Atención Primaria A rea III

C/Fuero, 18. Avilés.

33700 Asturitas.

Correo electrónico: arigueira@gapai03.insalud.es

\section{ABSTRACT}

\section{Set of Indicators for Assessing the Degree to which a Drug Therapy Guide is Followed in a primary care area}

Background: The constant gauging of drug prescription quality is part of the health-care process quality improvement programs as regards Primary Care, but, generally speaking, there are no tried and true indicators which can be used in this assessment process. Measuring the degree to which certain set drugs are adhered to as a district-wide guide regarding which a consensus of opinion has been reached can be used as an overall indicator of prescribing-related quality. The objective of this study is that of setting out a method for selecting quality indicators of a generalizing type, the same serving as an example with regard to selecting an indicator for gauging the degree to which the district guide is followed.

Methods: Descriptive cross-sectional study entailing the general practitioners in the Ifealth Care District of Avilés $(n=78)$ as the individual district analyzed, and the prescriptions written thereby which were invoiced in 1997 as the data source. Seven (7) indicators were prepared for gauging the degree to which the District Drug Therapy Guide was followed. A selection was made by studying the following aspects: reliability, ready availability, accuracy, sensitivity, specificity and coherence.

Results: The scores fell within the 10-23 range (out of a possible 28 ), the highest score being that of the percentage of prescriptions for active ingredients out of the guide in comparison to the total number of prescriptions written by each physician.

Conclusions: The indicators used in assessing drug-prescribing quality must first be analyzed in detail before being implemented. An innovative method is suggested for testing indicators for monitoring the prescribing of drugs as a whole. The most useful of all of the indicators studied for gauging the degree to which the Primary Care drug therapy guide was being followed is the percentage of prescriptions written for active ingredients out of the total number of prescriptions written. The degree to which the District Drug Therapy Guide is followed is related positively to quality prescribing.

Key words: Drug Therapy Guide. Form. Primary Care. Prescribing. Medications. Quality Control. Quality indicators. Assessment methodology. 


\section{INTRODUCCIÓN}

La evaluación de la calidad de prescripción farmacéutica es un proceso que puede estar integrado en el conjunto de evaluación de la calidad asistencial y que se caracteriza, en general, por la monitorización continua en el tiempo de indicadores de calidad de prescripción ${ }^{1}$. La utilización de indicadores de calidad de prescripción como herramienta para la identificación de problemas en la misma es un método indiscutible en la actualidad. Algunos autores distinguen entre «indicadores específicos», es decir, que parten de la medición de uso de medicamentos en un problema de salud concreto, e «indicadores genéricos o globalizadores», que califican el total de la prescripción utilizando clasificaciones previas de los medicamentos en función de una serie de características, como la utilidad terapéutica o el valor intrín$\mathrm{seco}^{2}$. Debido a problemas en la disponibilidad de la información necesaria para la construcción de indicadores específicos, en la mayoría de los sistemas sanitarios se ha preferido utilizar indicadores globalizadores como primer escalón ya que, pese a los problemas que puedan presentar ${ }^{3}$, pueden proporcionar un instrumento adecuado para el abordaje de actuaciones prioritarias. El objetivo de la monitorización de este tipo de indicadores, por tanto, sería el de observar su variación, de modo que se puedan identificar situaciones de «alerta» cuando el valor del indicador estudiado sobrepase un valor considerado inaceptable, permitiendo entonces la propuesta y puesta en práctica en tiempo real de intervenciones con finalidad correctora, manteniéndose simultáneamente la monitorización del indicador con objeto de conocer el efecto de las medidas implantadas1. Algunos autores incluso proponen escalonamientos de los indicadores de prescripción en varios niveles con objeto de obtener criterios para priorizar intervenciones, siendo el nivel más básico el de los indicadores globalizadores ${ }^{4,5}$. La experiencia del Instituto Nacional de la Salud (Insalud), hasta el momento, es amplia en la identificación de problemas de baja ca- lidad global, situaciones donde las actividades correctoras se han considerado prioritarias. Recientemente, se está planteando también la monitorización de la utilización de medicamentos de utilidad terapéutica alta ${ }^{6}$.

A pesar de una extensa revisión bibliográfica realizada, no se ha encontrado ninguna referencia hacia una metodología concreta para la selección de un indicador de calidad de prescripción global, hallándose, en cambio, referencias sobre el uso de ciertos indicadores de este tipo, sin un razonamiento específico que permita clarificar por qué se han utilizado esos en concreto y no otros que pudieran proponerse igualmente válidos a priori para realizar el mismo proceso de medida.

Una guía farmacológica, o formulario, se puede considerar como una selección de medicamentos, dentro del conjunto de una oferta disponible, para su utilización en un determinado contexto sanitario. De modo general, puede afirmarse que las guías farmacológicas persiguen la mejora cualitativa de los medicamentos utilizados, es decir, promover el uso de los fármacos de mejor relación beneficio-riesgo-coste de entre los disponibles en la oferta general, si bien este objetivo está supeditado a los criterios y forma de elaboración de estas guías. Podemos efectuar un seguimiento de adhesión al formulario utilizando un indicador genérico o globalizador, pudiendo resultar del mismo medidas correctoras de diversa índole (seguimiento de indicadores específicos para detectar problemas sanitarios concretos, abordaje de medidas educativas más intervencionistas, o la actualización de la guía...). La medida de la adhesión a las guías de atención primaria no se ha realizado de un modo rutinario y continuo en el conjunto de la facturación de las recetas médicas al Sistema Nacional de Salud, ni se han evaluado las consecuencias de la variación de dicha adhesión en nuestro ámbito sanitario. En este sentido, se debe aclarar que sí existen en la bibliografía estudios puntuales de seguimiento de alguna guía farmacológica ${ }^{7-10}$, ha- 
biéndose analizado incluso la reacción de facultativos y pacientes ante la introducción de un formulario ${ }^{11,12}$.

El Area III de Asturias (Avilés) cuenta con una Guía Farmacoterapéutica de Atención Primaria (AP) desde $1996^{13}$, elaborada de un modo participativo y consensuado con los profesionales de los equipos del Área. El proceso de selección se realizó partiendo de experiencias ya conocidas ${ }^{14,15}$, mediante la aplicación de un protocolo previo, en el que se priorizaron como criterios de inclusión, entre otros, las características de eficacia, seguridad y coste de los principios activos, por este orden ${ }^{16}$. Consta de una selección primaria de 387 principios activos, de cada uno de los cuales se detalla en el documento un número máximo de tres especialidades, las cuales no siempre coinciden con las más baratas del mercado.

El objetivo principal del presente estudio ha sido establecer un método para selección de indicadores de calidad de tipo globalizador. Para ello, y como objetivo secundario, se ha llevado a cabo la selección de un indicador destinado a medir, de un modo continuado, la adhesión de los médicos generales del área de Avilés a la Guía Farmacoterapéutica del Area.

\section{MATERIAL Y MÉTODOS}

Se realizó un estudio descriptivo transversal durante todo el año 1997, incluyendo como unidad de análisis todos los médicos generales de Atención Primaria del Área III Avilés (Asturias), asignados a un cupo de población. Se excluyó a cinco médicos generales que durante el periodo de estudio fueron coordinadores de equipo, porque en sus datos de prescripción figuraban, en un porcentaje alto, recetas utilizadas para atender poblaciones ajenas a su propio cupo, como recetas de médicos de refuerzo de fin de semana o verano. En total se incluyó a 78 médicos generales. Del conjunto de recetas facturadas de este colectivo durante el año
1997, se extrajo la batería de indicadores propuestos para medir la adhesión a la guía farmacoterapéutica que se detallan en la tabla 1 .

El método utilizado para la selección de un indicador es similar al método SOJA (System of Objectified Judgement Analysis) para selección de principios activos ${ }^{17,18}$. En nuestro caso, se ha planteado la valoración de una serie de criterios requeridos para los indicadores de calidad, intentando que dicha valoración sea objetiva, lo que no se ha conseguido para todos ellos, si bien se ha considerado que esto no invalidaría los resultados. La valoración de la selección se realizó sumando las puntuaciones obtenidas para cada una las siguientes características requeridas para los indicadores de calidad, según la bibliografía consultada ${ }^{1,2,19}$ : fiabilidad, facilidad de obtención, precisión, sensibilidad, especificidad y coherencia. Por tratarse de indicadores de tipo genérico concebidos para la medición continuada de la prescripción, es decir, utilizables en el proceso dinámico de evaluación, dichas características puntuaron del siguiente modo:

Fiabilidad: de valoración subjetiva en una escala de 0 a 3 , en función de la posibilidad de discrepancias en el momento de codificar los datos para su procesado y del número de transformaciones necesarias de los datos utilizados. En todos los indicadores en los que interviene el término envases, recetas o importe facturado mediante identificación previa de códigos nacionales de especialidad, no hay posibilidad de error en su identificación, existiendo, además, una correspondencia inequívoca con su inclusión o no en la guía (puntuación: 3 ). Se ha dado menor puntuación a los indicadores que cuantifican el número de principios activos mediante código ATC (2 puntos) o el número de DDDs ( 1 punto), ya que las características del mercado español han obligado a una clasificación extra en los medicamentos no contemplados por el WHO Collaborating Centre for Drug Statistis Methodology ${ }^{20}$, y dicha clasificación podria diferir de otras similares 
Tabla 1

Indicadores de adhesión a la guía estudiados

\begin{tabular}{|c|c|c|c|c|}
\hline INDICADOR & NUMERADOR & DENOMINADOR & EXPRESIÓN & FÓRMULA \\
\hline 11 & $\begin{array}{c}\text { Número de recetas facturadas } \\
\text { de todos los principios activos } \\
\text { incluidos en la guía (a) }\end{array}$ & $\begin{array}{l}\text { Número de recetas } \\
\text { totales facturadas (b) }\end{array}$ & Porcentaje & $\mathrm{a} / \mathrm{b}$ \\
\hline 12 & $\begin{array}{l}\text { Importe (P.V.P.) de las recetas } \\
\text { facturadas todos los principios } \\
\text { activos incluidos en la guía (a) }\end{array}$ & $\begin{array}{l}\text { Importe total } \\
\text { facturado (b) }\end{array}$ & Porcentaje & $\mathrm{a} / \mathrm{b}$ \\
\hline 13 & $\begin{array}{l}\text { Envases facturados de todos } \\
\text { los principios activos de la guía } \\
\text { por población activa (con } \\
\text { aportación) (al) y pasiva (sin } \\
\text { aportación) (a2) }\end{array}$ & $\begin{array}{l}\text { Población con } \\
\text { portación (p1) y sin } \\
\text { aportación }(\mathrm{p} 2), \text { meses } \\
\text { de facturación ( } \mathrm{t})\end{array}$ & $\begin{array}{l}\text { Envases por } 100 \\
\text { personas ajustado } \\
\text { por proporción de } \\
\text { con y sin aportación }\end{array}$ & $\begin{array}{c}\left(\left(\left(\mathrm{a} 1 /\left(\mathrm{p} 1^{*} \mathrm{t}\right)\right) *\right.\right. \\
0,7374)+(\mathrm{a} 2 /(\mathrm{p} 2 * \mathrm{t})) \\
* 0,2626))^{* 100}\end{array}$ \\
\hline 14 & $\begin{array}{l}\text { Número de recetas facturadas } \\
\text { de todas las especialidades } \\
\text { detalladas en la guia (a) }\end{array}$ & $\begin{array}{l}\text { Número de recetas } \\
\text { totales prescritas }(b)\end{array}$ & Porcentaje & $\mathrm{a} / \mathrm{b}$ \\
\hline 15 & $\begin{array}{l}\text { Número de principios activos } \\
\text { de la guía prescritos(a) }\end{array}$ & $\begin{array}{l}\text { Número de principios } \\
\text { activos distintos } \\
\text { prescritos(b) }\end{array}$ & Porcentaje & $\mathrm{a} / \mathrm{b}$ \\
\hline 16 & $\begin{array}{l}\text { Número de envases facturados } \\
\text { de principios activos incluidos } \\
\text { en la guía para subgrupos } \\
\text { A02B1(a1), B03A I(a2), } \\
\text { C02E(a3), C01D2(a4), } \\
\text { J03B(a5), M01A1(a6), } \\
\text { N06A3(a7), R06A 1(a8)* }\end{array}$ & $\begin{array}{c}\text { Número de envases } \\
\text { totales facturados } \\
\text { para los subgrupos } \\
\text { A02Bl(b1), B03A(b2), } \\
\text { C02E(b3), C01D2(b4), } \\
\text { J03B(b5), M01A1(b6), } \\
\text { N06A3(b7),R06A1(b8)* }\end{array}$ & Suma de porcentajes & $\begin{array}{l}(a 1 / b 1)+(a 2 / b 2)+ \\
(a 3 / b 3)+(a 4 / b 4)+ \\
(a 5 / b 5)+(a 6 / b 6)+ \\
(a 7 / b 7)+(a 8 / b 8)\end{array}$ \\
\hline 17 & $\begin{array}{l}\text { Número de DDD facturadas de } \\
\text { principios activos incluidos de } \\
\text { la guía para subgrupos } \\
\text { A02B1(a1), B03Al(a2), } \\
\text { C02E(a3), C01D2(a4), } \\
\text { J03B(a5), M01A1(a6), } \\
\text { N06A3(a7), R06A1(a8)* }\end{array}$ & $\begin{array}{c}\text { Número de DDD } \\
\text { totales facturadas } \\
\text { para los subgrupos } \\
\text { A02B1(b1), B03A(b2), } \\
\text { C02E(b3), C01D2(b4), } \\
\text { J03B(b5), M01A1(b6), } \\
\text { N06A3(b7), R06Al(b8)* }\end{array}$ & Suma de porcentajes & $\begin{array}{c}(\mathrm{a} 1 / \mathrm{b} 1)+(\mathrm{a} 2 / \mathrm{b} 2)+ \\
(\mathrm{a} 3 / \mathrm{b} 3)+(\mathrm{a} 4 / \mathrm{b} 4)+ \\
(\mathrm{a} 5 / \mathrm{b} 5)+(\mathrm{a} 6 / \mathrm{b} 6)+ \\
(\mathrm{a} / \mathrm{b} 7)+(\mathrm{a} 8 / \mathrm{b} 8)\end{array}$ \\
\hline
\end{tabular}

P.V.P.: precio venta al público, DDD: dosis diarias definidas.

* Denominación de subgrupos: A02B1 («Antihistamínicos H2»), B03Al («Hierro sólo»), C02E («Inhibidores del sistema renina angiotensin«»), C01D2 («Antagonistas de calcio»), J03B («Otros quimioterápicos»), M01A1 («Antiinflamatorios y antirreumáticos no esteroideos solos»), N06A3 («Otros antidepresivos») y R06A («Antihistamínicos sistémicos»).

realizadas, por ejemplo la del Sistema Andaluz de Salud. También recibió dos puntos el indicador que utiliza población en su fórmula, por la posibilidad de discrepancia en la selección del tipo de la misma (usuarios globales, sólo receptores de medicación...).

Facilidad de obtención: se valoró de un modo objetivo en una escala de 0 a 5 , teniendo en cuenta los medios disponibles actualmente y el tiempo empleado en el cálculo del indica- dor. Se consideró puntuación máxima (5) en el caso de una obtención del indicador absolutamente automatizada, tanto en lo referente a los datos de origen como al cálculo del indicador. Se restó un punto a dicha puntuación máxima por cada una de las siguientes características: requiere algún tipo de actualización manual, requiere consulta bibliográfica y/o codificación, el procedimiento automatizado al efecto sólo calcula numeradores y denominadores (o sea, el cálculo fi- 
nal es manual), el procedimiento informático no es específico sino que se basa en otras herramientas informáticas (MSOffice). En el caso de elaboración totalmente manual la puntuación sería cero.

Precisión: para valorar este concepto, se sumó la puntuación resultante de valorar cada uno de estos criterios: capacidad de cuantificar en sí cada acto de prescripción, en lugar de utilizar otros datos relacionados (1 punto); que tomen como unidad de medida del volumen de prescripción o facturación la unidad de dosificación o DDD (2 puntos); que se basen en unidades no económicas de medida y distintas a la DDD (recetas o envases) (1 punto); que presenten información del global de la facturación o prescripción de cada médico (1 punto); que contengan información de la totalidad de los principios activos seleccionados en la guía (1 punto). El rango de valores oscila, pues, entre 0 y 5 puntos.

La sensibilidad: capacidad del indicador para detectar problemas de calidad reales ${ }^{1}$, en este caso, capacidad de reflejar en el tiempo una disminución en la adhesión de la guía. Esta característica se valoró calculando el tiempo (meses) necesario para detectar el descenso en una desviación estándar (SD) del valor del indicador considerado en un momento dado. Se tomó como situación de partida la media mensual de los datos de los médicos del estudio y se realizó una simulación temporal de mantenimiento de prescripción con características cualitativas y cuantitativas similares a las previas, pero suponiendo que ninguno de los medicamentos prescritos después de la situación inicial estuviera incluido en el formulario, por lo que, para la mayoría de los indicadores, las nuevas prescripciones solo afectarían al denominador. Se dio puntuación máxima (5 puntos) cuando, para conseguir la disminución del valor del indicador en una SD, era suficiente un mes (puesto que los datos analizados tienen una cadencia mínima mensual). Por cada mes adicional necesario se restó un punto a dicho máximo, de modo que para un periodo superior a 5 meses la puntuación fue cero.

Especificidad: capacidad del indicador para excluir casos sin problemas ${ }^{1}$, en este caso, capacidad de reflejar variaciones en el sentido de aumento en la adhesión a la guía. Para calcular la especificidad se partió de la misma situación hipotética que para el caso de la sensibilidad, la simulación temporal se hizo suponiendo que todas las nuevas prescripciones realizadas serían fármacos pertenecientes a la guía y modificarían, por ello, tanto el numerador como el denominador de casi todos los indicadores. Así pues, la especificidad se midió como el tiempo (meses) necesario para detectar el aumento en una desviación estándar del valor del indicador. La puntuación se realizó como en el caso de la sensibilidad.

Coherencia: ésta es una característica que se ha definido de un modo particular para el indicador de adhesión a una guía, y consistiría en el grado de relación del indicador con los objetivos de la misma. De acuerdo con lo expuesto anteriormente, un indicador que mide la adhesión a las mismas debería estar relacionado con el nivcl de eficiencia y eficacia de la farmacoterapia utilizada (relación beneficio-riesgo-coste favorable) y, a la vez, debe estar mínimamente influido por circunstancias estructurales - no modificablestanto las del entorno como las personales del facultativo. Para analizar csta característica se estudiaron estadísticamente los patrones de variación de cada indicador con relación a una serie de variables que, según la revisión bibliográfica realizada ${ }^{21-27}$, podrían condicionar las variaciones de prescripción farmacológica en AP. Dichas variables se clasificaron en 3 bloques: relacionadas con la eficiencia y/o la eficacia de la prescripción (bloque 1 o B1), mixtas (pueden medir el nivel de eficiencia y/o eficacia, pero son también estructurales) (bloque 2 o B2), estructurales (bloque 3 o B3).

El análisis estadístico incluyó en primer lugar una matriz de correlación entre variables y grado de significación bilateral de di- 
chas correlaciones. Posteriormente se efectuaron regresiones lineales multivariantes en bloques con finalidad descriptiva, tomando cada uno de los indicadores de adhesión a la guía como variable dependiente y todas las demás - excepto gasto, debido a la alta colinearidad obtenida con respecto a la variable población-, como independientes. Se obtuvieron las razones de los coeficientes de determinación múltiple $\left(\mathrm{R}^{2}\right)$ del bloque 1 y del conjunto de los bloques 1 y 2 sobre el total del coeficiente de determinación de los 3 bloques $\left(\mathrm{R}^{2}[\mathrm{~B} 1] / \mathrm{R}^{2}[\mathrm{~B} 1-\mathrm{B} 2-\mathrm{B} 3]\right)$ y $\mathrm{R}^{2}[\mathrm{~B} 1-$ $\mathrm{B} 2]$ / R2 [B1-B2-B3], respectivamente). De este modo, se pretendía conocer qué porcentaje de explicación de la variabilidad del indicador tendría cada uno de los bloques (B1 o B1+B2) sobre el global de la explicación conseguida utilizando todas las variables $(\mathrm{B} 1+\mathrm{B} 2+\mathrm{B} 3)$.

La característica coherencia se puntuó del siguiente modo en función de los resultados del análisis (con un máximo de hasta 5 puntos):

- Si el signo del coeficiente de la correlación simple entre los indicadores y todas las variables del bloque 1 (B1) es negativo y significativo, se otorgó un punto. En el caso de que todos los signos de correlación con $\mathrm{B} 1$ fueran positivos, la característica coherencia se puntuó como cero definitivamente, puesto que significaría que el incremento del indicador se relacionaría con alto gasto y baja calidad de prescripción farmacológica global.

- $\quad \mathrm{Si} \mathrm{R}^{2}[\mathrm{~B} 1] / \mathrm{R}^{2}[\mathrm{~B} 1-\mathrm{B} 2-\mathrm{B} 3]>50 \%$, se otorgó 1 punto.

- $\quad \mathrm{Si} \mathrm{R}^{2}$ [B 1-B2]/ $\mathrm{R}^{2}$ [B1-B2-B3] $>75 \%$, se valoró con 1 punto.

- Finalmente, se dio 1 punto por cada variable de los bloques 1 ó 2 que fuera significativa en el modelo global y que, además, tuviera un valor de coeficiente estandarizado - betasuperior al de cualquicra de las va- riables seleccionadas del bloque 3 . En este caso, se estarían excluyendo variables estructurales - no susceptibles de modificación - con una participación importante en la explicación de la variabilidad del indicador.

Herramientas utilizadas. Bases de datos (todas en $\mathrm{dBaseIV}$ ): códigos nacionales de especialidad (c.n.e.) facturados por facultativo mensualmente (aplicación Sistema de Información Farmacéutico de Asturias - SIFAS-); c.n.e. de los principios activos y de la selección de especialidades contenidas en la guía (elaboración propia y actualización mensual de modo manual); asignación de código ATC y DDD para los c.n.e. de principios activos contemplados por del WHO Collaborating Centre for Drug Statistics Methodology $\mathrm{y}^{20}$ junto con una codificación y asignación de DDD complementaria para principios activos no contemplados en la misma (elaborada y actualizada manualmente en la Dirección Territorial del Insalud de Zaragoza). Programas: de elaboración propia en dBaseIV para realizar el cruce de la base de datos de la aplicación SIFAS con la de c.n.e. de principios activos (I1, I2, I3) y de especialidades de la guía (14) respectivamente; gestor de base de datos (ACCESS) para elaborar los indicadores I5, I6 e I7; paquete estadístico SPSS/PC+ v. 6.1; herramienta «Solver» de Excel 97 para resolución de ecuaciones.

\section{RESULTADOS}

Fiabilidad: los resultados se reflejan en la segunda columna de la tabla 6 .

Facilidad de obtención: de acuerdo con las herramientas utilizadas ningún indicador recibió la máxima puntuación. Los resultados figuran en la tercera columna de la tabla 6 .

Precisión: ninguno de ellos obtuvo máxima puntuación, por no disponer de datos de la medicación realmentc prescrita, ya que todos utilizan datos de facturación. La puntuación figura en la cuarta columna de la tabla 6 . 
Análisis de sensibilidad y especificidad: Los datos e indicadores medios de la tabla 2 representan la situación hipotética de partida, que corresponderían a la atención de una población media de 1.180 acti- vos y 537 pensionistas. En la misma se especifican, además, el resto de los descriptivos principales. Con estos indicadores se han calculado las variaciones necesarias en el denominador, para el caso de determinación de

Tabla 2

Descriptivos principales de los indicadores de otras variables del estudio y de las características de prescripción (datos anuales)

\begin{tabular}{|c|c|c|c|c|c|c|}
\hline & MEDIA & MÍNIMO & MÁXIMO & DESV. TIPICA & VARIANZA & RANGO \\
\hline I1 $(1,2)$ & 0,604 & 0,509 & 0,698 & 0,037 & 0,001 & 0,190 \\
\hline $12(1.2)$ & 0,517 & 0,413 & 0,619 & 0,045 & 0,002 & 0,206 \\
\hline $\mathrm{I} 3(1)$ & 60,71 & 36,77 & 76,24 & 7,74 & 59,93 & 39.47 \\
\hline I4 (1,2) & 0,399 & 0,311 & 0,471 & 0,033 & 0,001 & 0,159 \\
\hline $15(1,2)$ & 0,362 & 0,325 & 0,401 & 0,018 & 0,0003 & 0,035 \\
\hline $16(1)$ & 1,583 & 0,527 & 2.759 & 0,536 & 0,287 & 2,233 \\
\hline $17(1)$ & 3,687 & 2,843 & 4,593 & 0,387 & 0,150 & 1,750 \\
\hline$N^{\circ}$ RECETAS (1) & 21.808 & 9.228 & 35.859 & 4.819 & 23.224 .918 & 26.631 \\
\hline $\mathrm{N}^{\circ}$ ENVASES $(1,3)$ & 22.393 & 9.560 & 36.869 & 4.929 & 24.296 .498 & 27.309 \\
\hline IMPORTE $(1)$ & 39.107 .817 & 18.057 .280 & 66.502 .850 & $8.871,429$ & $7,87 \mathrm{e}^{13}$ & 48.445 .570 \\
\hline$N^{n}$ de P. $\Lambda .(1)$ & 651 & 542 & 773 & 51 & 2.564 & 231 \\
\hline GASTO (1) & 34.591 .807 & 15.129 .467 & 59.754 .927 & 8.058 .510 & $6,5 \mathrm{e}^{13}$ & 44.625 .460 \\
\hline GASTO/P (1) & $1.493,9$ & 999,4 & $2.466,7$ & 219,2 & $48.050,2$ & $1.467,3$ \\
\hline EAP & & NO $(19 \%)$ & SÍ $(81 \%)$ & & & \\
\hline RURAL & & Sí (12\%) & NO $(88 \%)$ & & & \\
\hline TURISMO & & Sí (22\%) & $\mathrm{NO}(78 \%)$ & & & \\
\hline COMPROMISO & & NO $(28 \%)$ & SÍ $(72 \%)$ & & & \\
\hline ESPECIALIDAD & & Sİ (37\%) & $\mathrm{NO}(63 \%)$ & & & \\
\hline CONTINUIDAD & & SÍ (47\%) & NO $(53 \%)$ & & & \\
\hline DOCENCIA & & SÍ (12\%) & $\mathrm{NO}(88 \%)$ & & & \\
\hline PARTICIPACIÓN & & SÍ (14\%) & $\mathrm{NO}(86 \%)$ & & & \\
\hline SEXO & & MUJER (26\%) & VARÓN (74\%) & & & \\
\hline $\mathrm{EDAD}$ & 42 & 30 & 64 & 7,28 & 53,00 & 34 \\
\hline POBL. TOTAL (1) & 1.717 & 824 & 2.275 & 319,09 & $101.821,08$ & 1.451 \\
\hline POBL. $65(1)$ & 340 & 164 & 567 & 91,71 & $8.410,35$ & 403 \\
\hline CRONICOS (1) & 0,477 & 0,370 & 0,611 & 0,048 & 0,002 & 0,241 \\
\hline UTB (1) & 12,89 & 5,66 & 22,95 & 3,660 & 13,394 & 17,290 \\
\hline
\end{tabular}

$N^{\circ}$ : número, P.A.: Principios activos distintos según el criterio de principio activo considerado — ver texto-.

(1): Distribución normal (prueba Kolmogorov-Smirnov). (2): Expresado en tanto por uno. (3): el 32,24\% son de activos y los porcentajes correspondientes a los subgrupos A02B1, B03A, C02E, C02D1, J03B, M01 A1. N06A3 y R06A son, respectivamente: 2,10\%, $1,07 \%, 2,83 \%, 3,49 \%, 0,43 \%, 5,29 \%, 1,82 \%$ y $1,40 \%$.

Nomenclatura: GASTO: gisto farmacéutico, GASTO/P; Gasto/persona protegida ajustado, EAP: modelo asistencial reformado, RURAL: entorno rural, TURISMO: incidencia turística, COMPROMISO: existencia de compromiso de gestión, ESPECIALIDAD: formación postgrado especializada, CONTINUIDAD: estabilidad en puesto de trabajo, DOCENCIA: tutoría de docencia PARTICIPACIÓN: participación en comisiones, POBLACION: cupo de población total, Pobl. 65: porcentaje de población mayor de 65 años, CRÓNICOS: porcentaje de importe (P.V.P.) de medicación de aportación reducida y TLD - tratamiento de larga duración - sobre total de importe, UTB: indicador de baja calidad de prescripción o envases de baja calidad terapéutica/ 100 personas protegidas ajustado. 
sensibilidad, y del numerador y denominador, en la determinación de la especificidad (4a columna de la tabla 3 ). El número de meses estimado (teniendo en cuenta cuando proceda las representaciones de las figuras 1 y 2) para obtener dichas variaciones, figura en la $5^{\mathrm{a}}$ columna de la misma tabla.

Tabla 3

Datos del análisis de la sensibilidad (S) y de la especificidad (E)

\begin{tabular}{|c|c|c|c|c|c|}
\hline $\begin{array}{l}I N D I- \\
A D O R\end{array}$ & $\begin{array}{c}\text { VALOR INICIAL } \\
\text { DE NUMERADORES } \\
\text { Y DENOMINADORES }\end{array}$ & ECUACIÓN & $\begin{array}{c}\text { RESULTADOS } \\
\text { DE LA } \\
\text { ECUACION * }\end{array}$ & $\begin{array}{c}\text { TIEMPO } \\
\text { ESTIMADO } \\
\text { NECESARIO } \\
\text { PARA OBTENER } \\
\text { ELRESULTADO }\end{array}$ & $\begin{array}{c}\text { COMENTARIO } \\
\text { ALA } \\
\text { ESTIMACIÓN } \\
\text { DELTIEMPO }\end{array}$ \\
\hline Il & $\begin{array}{l}\mathrm{a}=13.172, \\
\mathrm{~b}=21.808\end{array}$ & $\begin{array}{c}\text { S: }: a(b+x)=0,5673(1) \\
\text { E: }(a+x) /(b+x)=0,6407(1)\end{array}$ & $\begin{array}{l}x=1.411 \\
x=2.228\end{array}$ & $\begin{array}{c}1 \text { mes } \\
2 \text { meses }\end{array}$ & $\begin{array}{l}\text { El crecimiento en } n^{\circ} \\
\text { de recetas acumulado } \\
\text { es lineal (ver fig. 1) }\end{array}$ \\
\hline 12 & $\begin{array}{l}a=20.230 . .507 \\
b=39.107 .817\end{array}$ & $\begin{array}{c}\text { S: } a /(b+x)=0,4726(1) \\
E:(a+x) /(b+x)=0,5621(1)\end{array}$ & $\begin{array}{l}x=3.699 .006 \\
x=4.000 .984\end{array}$ & $\begin{array}{l}2 \text { meses } \\
2 \text { meses }\end{array}$ & $\begin{array}{l}\text { El crecimiento en } \\
\text { importe acumulado } \\
\text { es lineal (ver fig. 1) }\end{array}$ \\
\hline 13 & $\begin{array}{c}\mathrm{a}_{1}=4.468 \\
\mathrm{a}_{2}=9.183 \\
\mathrm{p}_{1}=1.180 \\
\mathrm{p}_{2}=537 \\
\mathrm{t}=12\end{array}$ & $\begin{array}{c}\text { S: }\left(\left(\left(\mathrm{a} 1 /\left(\mathrm{p} 1^{*}(\mathrm{t}+\mathrm{x})\right) *\right.\right.\right. \\
0,7374)+(\mathrm{a} 2 /(\mathrm{p} 2 *(\mathrm{t}+\mathrm{x}))) \\
* 0,2626))^{*} 100=52,97(1) \\
\mathrm{E}:\left(\left(\left((\mathrm{al}+\mathrm{x} 1) /\left(\mathrm{pl} \mathrm{l}^{*}(\mathrm{t}+\mathrm{x})\right)\right.\right.\right. \\
* 0,7374)+((\mathrm{a} 2+\mathrm{x} 2) / \\
(\mathrm{p} 2 *(\mathrm{t}+\mathrm{x}))) * \\
0,2626))^{*} 100=68,45(1)\end{array}$ & $\begin{array}{c}x=1,75 \\
x_{1}=1.561 \\
x_{2}=3.243 \\
x=2,3\end{array}$ & $\begin{array}{l}2 \text { meses } \\
3 \text { meses }\end{array}$ & \\
\hline 14 & $\begin{array}{l}a=8.675 \\
b=21.808\end{array}$ & $\begin{array}{c}\text { S: } a /(b+x)=0,3635(1) \\
E:(a+x) /(b+x)=0,4322(1)\end{array}$ & $\begin{array}{l}x=2.058 \\
x=1.321\end{array}$ & $\begin{array}{l}2 \text { meses } \\
1 \text { mes }\end{array}$ & $\begin{array}{l}\text { El crecimiento en } n^{\prime \prime} \\
\text { de recetas acumulado } \\
\text { es lineal (ver fig. 1) }\end{array}$ \\
\hline 15 & $a=311$, & $\mathbf{E}:(a+x) /(b+x)=0,4799(1)$ & $x=34$ & 8 meses & $\begin{array}{l}\text { El crecimiento de } \mathrm{n}^{\prime} \\
\text { de p. a. distintos } \\
\text { acumulados no es } \\
\text { lineal, se ha hecho } \\
\text { media con los datos } \\
\text { en crecimiento de los } \\
3 \text { últimos meses, } \\
\text { diferenciando entre } \\
\text { p.a. totales y de la } \\
\text { guía (ver fig. 2) }\end{array}$ \\
\hline 16 & $\begin{array}{c}a_{1}=123, a_{2}=81, \\
a_{3}=89, a_{4}=75, \\
a_{5}=16, a_{6}=28, \\
a_{7}=113, a_{8}=154, \\
b_{1}=393, b 2=232, \\
b_{3}=617, b 4=768, \\
b_{5}=90, b 6=1.149, \\
b_{7}=393, b 8=301\end{array}$ & $\begin{array}{c}\text { S: }(a 1 /(b 1+x 1))+\ldots+ \\
(a 8 /(b 8+x 8))=1,0471 \\
\\
\text { E: }((a 1+x 1) /(b 1+x 1))+ \\
\ldots+((a 8+x 8) /(b 8+x 8))= \\
2,1186\end{array}$ & $\begin{array}{c}x_{1}=227, x_{2}=115, \\
x_{3}=310, x 4=381, \\
x_{5}=43, x 6=562, \\
x_{7}=198, x 8=149 \\
x_{1}=41, x_{2}=21 \\
x_{3}=56, x 4=69, \\
x_{5}=8, x 6=102, \\
x_{7}=36, x 8=27\end{array}$ & 6 meses & $\begin{array}{l}\text { Se ha considerado la } \\
\text { media de prescripción } \\
\text { mensual de los } \\
\text { subgrupos con mayor } \\
\mathrm{n}^{\prime \prime} \text { de envases por mes } \\
\text { (M01Al) (ver fig. 1) }\end{array}$ \\
\hline 17 & $\begin{array}{c}\mathrm{a}_{1}=2.342, \mathrm{a}_{2}=2.374, \\
\mathrm{a}_{3}=19.689, \\
\mathrm{a}_{4}=17.047, \\
\mathrm{a}_{5}=92, \mathrm{a}_{6}=7.725, \\
\mathrm{a}_{7}=92, \mathrm{a}_{8}=4.751, \\
\mathrm{~b}_{1}=3.274, \mathrm{~b}_{2}=3.329, \\
\mathrm{~b}_{3}=22.398, \mathrm{~b}_{4}=26.574, \\
\mathrm{~b}_{5}=1.122, \mathrm{~b}_{6}=20.304 \\
\mathrm{~b}_{7}=10.749, \mathrm{~b}_{8}=4.751\end{array}$ & $\begin{array}{c}\text { S: }(a 1 /(b 1+x 1))+\ldots+ \\
(a 8 /(b 8+x 8))=3,0864 \\
\\
\text { E: }((a 1+x 1) /(b 1+x 1))+ \\
\ldots+((a 8+x 8) /(b 8+x 8))= \\
4,4117\end{array}$ & $\begin{array}{c}x_{1}=923, x_{2}=669, \\
x_{3}=4.461, x_{4}=5.366, \\
x_{5}=217, x_{6}=3.981 \\
x_{7}=2.176, x_{8}=957 \\
x_{1}=835, x_{2}=605, \\
x_{3}=4.035, x_{4}=4.854, \\
x_{5}=196, x_{6}=3.601, \\
x_{7}=1.968, x_{8}=865\end{array}$ & 3 meses & $\begin{array}{l}\text { Se ha considerado } \\
\text { la media de } \\
\text { prescripción mensual } \\
\text { de los subgrupos con } \\
\text { mayor n" de DDD } \\
\text { por mes (C02E) } \\
\text { (ver fig. 1) }\end{array}$ \\
\hline
\end{tabular}

*: Restricciones impuestas para la resolución de las ecuaciones: para todos los indicadores que los valores de incremento $\left(\mathrm{x}, \mathrm{x}_{1} \ldots\right) \geq$ 0 , además para 16 e 17 que la relación entre denominadores se mantuviera en la misma proporción que la de los datos de partida; en de la ecuación de especificidad para el indicador $\mathrm{I} 3$, que el incremento de $\mathrm{t}(\mathrm{x})$ fuera $\leq 6$ meses y que la relación envases pensionistas/envatses activos tuviera una variación máxima de $\pm 5 \%$ respecto al valor medio original. 
Figura 1

Evolución mensual de denominadores de I1, I2, I4, I6 y I7

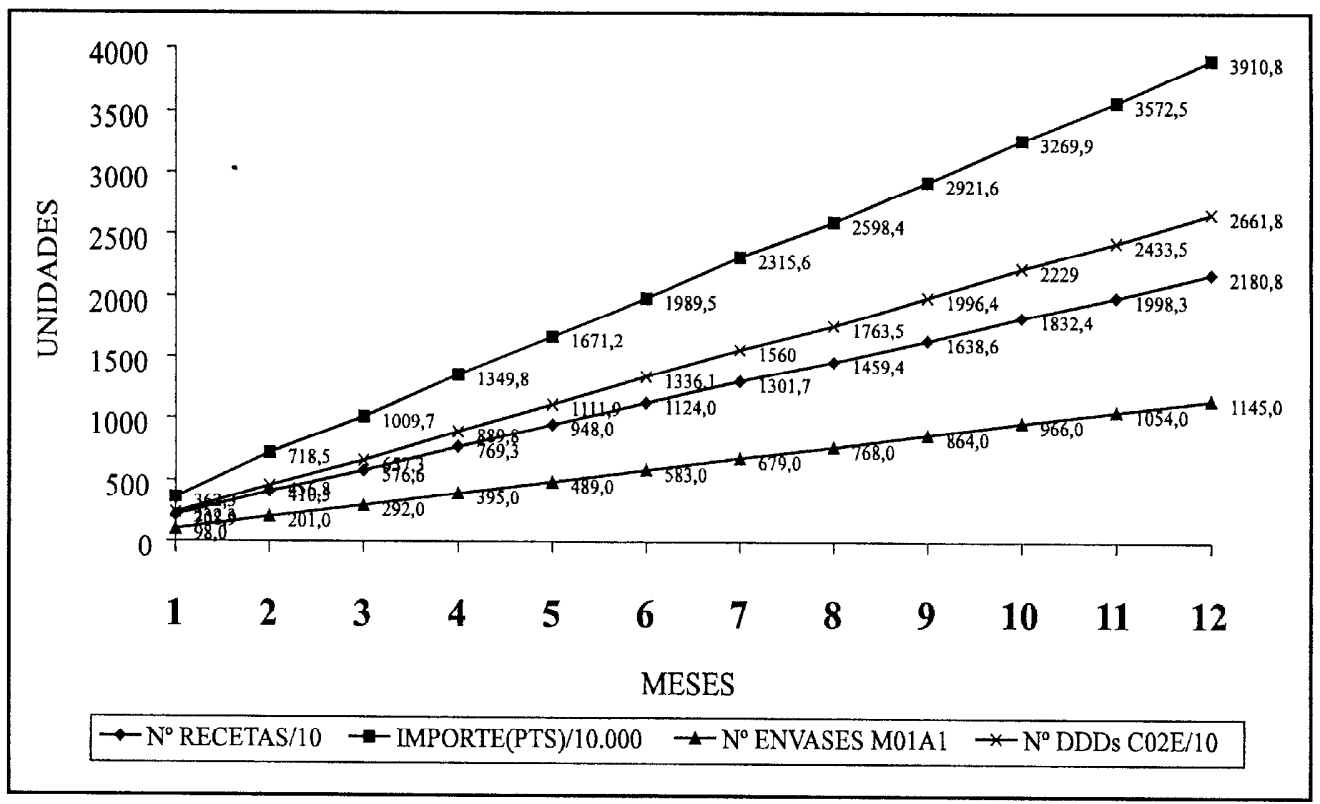

En cada mes se acumulan los datos de los meses anteriores.

Figura 2

Evolución mensual de numeradores y denominadores de I5

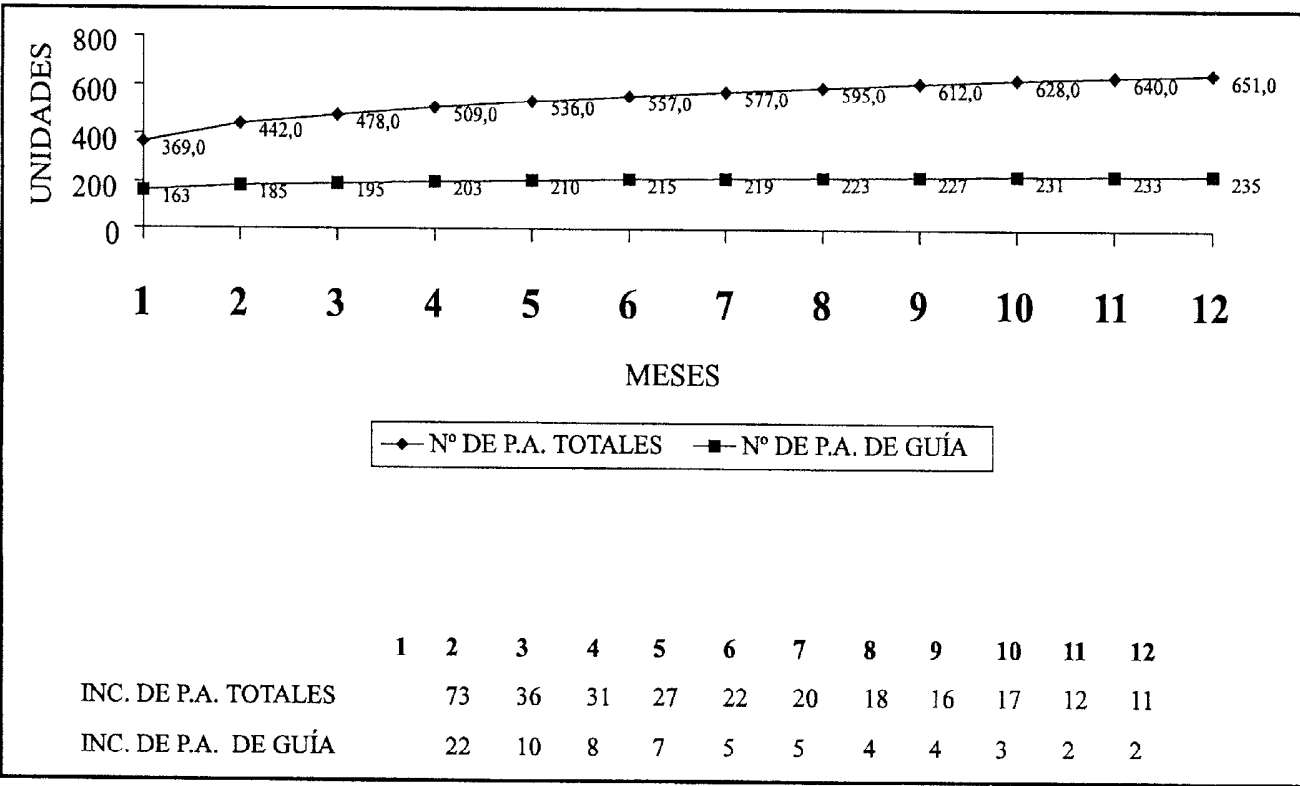

En cada mes se acumulan los datos de los meses anteriores. INC.: incremento, P.A.: principios activos. 
Análisis de la coherencia: En la Tabla 2 también se recogen los estadísticos principales de las variables analizadas en este análisis. Los coeficientes de correlaciones de las regresiones lineales bivariantes se observan en la tabla 4. Según éstos datos, y no teniendo en cuenta la posible confusión del resto de las variables, parece que los indicadores I1, I2, I4, I5 e 17 (I6 con menor fuerza) tienen un comportamiento muy similar, mientras que $\mathrm{I} 3$ difiere de los anteriores por sus correlaciones de signo distinto al del resto de los indicadores sobre todo con gasto, gasto/P y UTB.

Tabla 4

Correlaciones y significación entre variables

\begin{tabular}{|c|c|c|c|c|c|c|c|c|}
\hline & & 11 & 12 & 13 & 14 & 15 & 16 & 17 \\
\hline & 11 & 1.00 & & & & & & \\
\hline & 12 & $0,85(1)$ & 1.00 & & & & & \\
\hline & 13 & 0,15 & 0,21 & 1.00 & & & & \\
\hline & 14 & $0,73(1)$ & $0,64(1)$ & 0,03 & 1.00 & & & \\
\hline & 15 & $0,53(1)$ & $-42(1)$ & $-0,24(3)$ & $0,65(1)$ & 1.00 & & \\
\hline & 16 & $0,39(1)$ & $0,36(1)$ & $-0,02$ & $0,37(1)$ & 0,20 & 1.00 & \\
\hline & 17 & $0,67(1)$ & $0,65(1)$ & 0,17 & $0,62(1)$ & $0,32(2)$ & $0,37(1)$ & 1.00 \\
\hline \multirow{2}{*}{ BLOQUE 1} & GASTO/P & $-0,46(1)$ & $-0,27(2)$ & $0,70(1)$ & $-0,45(1)$ & $-0,47(1)$ & $-0,23(3)$ & $-0,24(3)$ \\
\hline & UTB & $-0,49(1)$ & $-0,28(3)$ & $0,48(1)$ & $-0,65(1)$ & $-0,72(1)$ & $-0,39(1)$ & $-0,49(1)$ \\
\hline \multirow{3}{*}{ BI OQUE 2} & GASTO & $-0,24(3)$ & $-0,10$ & $0,37(1)$ & $-0,34(2)$ & $-0,60(1)$ & $-0,02$ & $-0,09$ \\
\hline & COMPROMISO & $0,53(1)$ & $0,51(1)$ & 0,19 & $0,52(1)$ & $0,49(1)$ & 0,16 & $0,48(1)$ \\
\hline & CRÓNICOS & $0,56(1)$ & 0,20 & $-0,07$ & $0,56(1)$ & $0,49(1)$ & 0,21 & $0.40(1)$ \\
\hline \multirow{12}{*}{ BLOQUE 3} & CONTINUIDAD & 0,10 & 0,20 & $-0,02$ & $-0,01$ & 0.12 & 0,06 & 0,02 \\
\hline & DOCENCIA & $0,25(3)$ & $0,33(2)$ & 0,06 & $0,27(3)$ & $0,34(2)$ & 0,02 & $0,26(3)$ \\
\hline & EAP & $0,48(1)$ & $0,40(1)$ & 0,17 & $0,44(1)$ & $0,36(1)$ & $0,30(2)$ & $0,47(1)$ \\
\hline & $-E D A D$ & $-0,18$ & $-0,22(3)$ & $-0,24(3)$ & $-0,26(3)$ & $-0,32(2)$ & $-0,10$ & $-0,25(3)$ \\
\hline & ESPECIALIDAD & $0,34(2)$ & $0,26(3)$ & 0,02 & $0,34(3)$ & $0,44(1)$ & 0,11 & $0,24(3)$ \\
\hline & PARTICIPACION & 0,02 & 0,08 & $-0,11$ & 0,04 & 0,15 & $0,24(3)$ & 0,12 \\
\hline & POBLACIÓN & $-0,18$ & $-0,15$ & $-0,22(3)$ & $-0,26$ & $-0,33(2)$ & 0,04 & $-0,17$ \\
\hline & POBL. PENS & 0,13 & 0,13 & 0,05 & 0,01 & $-0,39(1)$ & 0,19 & 0,14 \\
\hline & POBL.65 & 0,13 & 0,14 & 0,05 & $-0,04$ & $-0,38(1)$ & 0,18 & 0,16 \\
\hline & RURAL & $0.23(3)$ & $0,25(3)$ & $-0,05$ & 0,12 & $-0,09$ & 0,04 & $0,30(2)$ \\
\hline & SEXO & 0,19 & 0,14 & $0,32(2)$ & 0,17 & 0,06 & 0,12 & 0,11 \\
\hline & TURISMO & 0,03 & $-0,11$ & $-0,03$ & $-0,01$ & $-0,03$ & $-0,07$ & $-0,00$ \\
\hline
\end{tabular}

(1): $p \leq 0,001,(2): p \leq 0.01 .(3): p \leq 0,05$ Resto: $p>0,05$.

El análisis estadístico de la regresión multivariante obtuvo como resultados los que se nan resumido en la tabla 5 y el resul- tado de la puntuación global de la coherencia figura en la penúltima columna de la tabla 6. 
Tabla 5

Análisis de coherencia de los indicadores. Regresión multivariante en bloques

\begin{tabular}{|c|c|c|c|c|c|c|c|c|}
\hline & ESTADÍSTICOS* & 11 & 12 & 13 & 14 & 15 & 16 & 17 \\
\hline \multirow{5}{*}{ 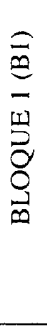 } & R Múltiple & 0,530 & 0,311 & 0,709 & 0,657 & 0,719 & 0,285 & 0,389 \\
\hline & $\mathrm{R}^{2}$ & 0,281 & 0,097 & 0,503 & 0,432 & 0,517 & 0,081 & 0,151 \\
\hline & Beta final UTB & $-0,0329$ & 0,1456 & $\begin{array}{c}0,4481 \\
\left(\mathrm{~S}_{\mathrm{T}}\right)\end{array}$ & $\begin{array}{c}-0,3867 \\
\left(\mathrm{~S}_{\mathrm{T}}\right)\end{array}$ & $\begin{array}{c}-0,4568 \\
(\mathrm{ST})\end{array}$ & $-0,1332$ & $-0,1118$ \\
\hline & Beta final GASTO/P & $\begin{array}{c}-0,3474 \\
\left(\mathrm{~S}_{\mathrm{T}}\right)\end{array}$ & $\begin{array}{c}-0,3927 \\
\left(\mathrm{~S}_{\mathrm{T}}\right)\end{array}$ & $\begin{array}{c}0,5997 \\
\left(\mathrm{~S}_{\mathrm{T}}\right)\end{array}$ & $\begin{array}{c}-0,1911 \\
\left(\mathrm{~S}_{\mathrm{T}}\right)\end{array}$ & $\begin{array}{c}-0,1799 \\
\left(\mathrm{~S}_{\mathrm{T}}\right)\end{array}$ & $-0,1016$ & $-0,0951$ \\
\hline & $\begin{array}{l}\mathrm{F} \\
\text { (sig. F) }\end{array}$ & $\begin{array}{l}14.679 \\
(<001)\end{array}$ & $\begin{array}{l}4,010 \\
(0,022)\end{array}$ & $\begin{array}{l}37,895 \\
(<001)\end{array}$ & $\begin{array}{l}28,462 \\
(<001)\end{array}$ & $\begin{array}{l}40,199 \\
(<001)\end{array}$ & $\begin{array}{c}3,313 \\
(0,042)\end{array}$ & $\begin{array}{c}6,688 \\
(0,002)\end{array}$ \\
\hline \multirow{5}{*}{ 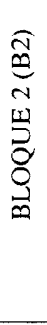 } & R Múltiple & 0,680 & 0,576 & 0,836 & 0,717 & 0,744 & 0,293 & 0,529 \\
\hline & $\mathrm{R}^{2}$ & 0,462 & 0,332 & 0,671 & 0,514 & 0,554 & 0,086 & 0,279 \\
\hline & $\begin{array}{l}\text { Beta final } \\
\text { COMPROMISO }\end{array}$ & $\begin{array}{l}0,3568 \\
\left(\mathrm{~S}_{\mathrm{T}}\right)\end{array}$ & $\begin{array}{c}0,6115 \\
\left(\mathrm{~S}_{\mathrm{T}}\right)\end{array}$ & $\begin{array}{l}0,3668 \\
\left(\mathrm{~S}_{\mathrm{T}}\right)\end{array}$ & $\begin{array}{l}0,1948 \\
\left(\mathrm{~S}_{\mathrm{T}}\right)\end{array}$ & $\begin{array}{c}0,2715 \\
\left(\mathrm{~S}_{\mathrm{T}}\right)\end{array}$ & $-0,2349$ & $\begin{array}{l}0,3130 \\
\left(\mathrm{~S}_{\mathrm{T}}\right)\end{array}$ \\
\hline & $\begin{array}{l}\text { Beta final } \\
\text { CRONICOS }\end{array}$ & 0,1722 & $-0,2331$ & 0,1679 & 0,1620 & $-0,0122$ & 0,0931 & 0,1585 \\
\hline & $\begin{array}{l}F \\
\text { (sig. F) } \\
\end{array}$ & $\begin{array}{c}15,657 \\
(<0,001) \\
\end{array}$ & $\begin{array}{r}9,056 \\
(<0,001) \\
\end{array}$ & $\begin{array}{c}37,294 \\
(<0,001) \\
\end{array}$ & $\begin{array}{c}19,287 \\
(<0,001) \\
\end{array}$ & $\begin{array}{c}22,640 \\
(<0,001)\end{array}$ & $\begin{array}{r}1,712 \\
(0,157) \\
\end{array}$ & $\begin{array}{c}7,074 \\
(<0,001)\end{array}$ \\
\hline \multirow{6}{*}{ 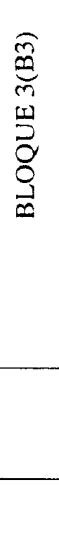 } & R Múltiple & 0,707 & 0,620 & 0,836 & 0,799 & 0,8385 & 0,391 & 0,616 \\
\hline & $\mathrm{R}^{2}$ & 0,500 & 0,384 & 0,699 & 0,638 & 0,703 & 0,153 & 0,379 \\
\hline & $\begin{array}{l}\text { VARIABLES } \\
\text { SELECCIONADAS } \\
\text { con } \mathrm{S}_{\mathrm{T}} \\
\text { (Beta) }\end{array}$ & $\begin{array}{c}\text { SEXO } \\
(0,2052)\end{array}$ & $\begin{array}{l}\text { EDAD } \\
(-0,2477)\end{array}$ & $\begin{array}{l}\text { ESPECIA- } \\
\text { LIDAD } \\
(0,1752)\end{array}$ & $\begin{array}{c}\text { SEXO } \\
(0,2137), \\
\text { P>65 } \\
(0,2989), \\
\text { TURISMO } \\
(\sim 0,2733)\end{array}$ & $\begin{array}{c}\text { POBLA- } \\
\text { CIÓN } \\
(-0,4100) \\
\text { P>65 } \\
(-0,3141)\end{array}$ & $\begin{array}{c}\text { EAP } \\
(0,3683)\end{array}$ & $\begin{array}{c}\text { P>65 } \\
(0,3307), \\
\text { TURIS- } \\
\text { MO } \\
(-0,2844)\end{array}$ \\
\hline & $F(\operatorname{sig} . F)$ & $\begin{array}{c}14,397 \\
(<0,001)\end{array}$ & $\begin{array}{c}8,975 \\
(<0,001)\end{array}$ & $\begin{array}{c}33,494 \\
(<0,001)\end{array}$ & $\begin{array}{c}17,622 \\
(<0,001)\end{array}$ & $\begin{array}{c}28,021 \\
(<0,001)\end{array}$ & $\begin{array}{c}2,602 \\
(0,032)\end{array}$ & $\begin{array}{c}7,230 \\
(<0,001)\end{array}$ \\
\hline & $\begin{array}{l}\text { Razón } R^{2}(\mathrm{~B} 1 \text { y } \mathrm{B} 2) / \\
\mathrm{R}^{2}(\mathrm{~B} 1, \mathrm{~B} 2 \text { y } \\
\mathrm{B} 3)^{*} 100\end{array}$ & $92,4 \%$ & $86,5 \%$ & $96,0 \%$ & $80,6 \%$ & $78,9 \%$ & $6,2 \%$ & $73,6 \%$ \\
\hline & $\begin{array}{l}\text { Razón } \mathrm{R}^{2} \text { (B 1)/ } \mathrm{R}^{2} \\
(\mathrm{~B} 1, \mathrm{~B} 2 \text { y B } 3)^{*} 100\end{array}$ & $56,2 \%$ & $25,3 \%$ & $71,9 \%$ & $67,7 \%$ & $73,5 \%$ & $52,9 \%$ & $39,8 \%$ \\
\hline
\end{tabular}

(ST) Significación de T,05 en modelo final, Sig.: significación.

Ohtenidos por modelo de regresión múltiple en bloques obligándose primero la entrada de las variables del bloque 1, tuego las del bloque 2, utilizando en ambos casos el método «Enter», y finalmente se realizó la entrada de las variables del bloque 3 con el método de selección por «Pasos sucesivos».

Según los resultados globales reflejados en la tabla 6, el indicador con mayor puntuación, y por tanto, preferente en principio para la selección buscada, sería el Il mientras que I6 e 17 serían los menos adecuados para un seguimiento global de la guía

\section{DISCUSIÓN}

Son escasas las publicaciones relacionadas con la metodología de la evaluación de la calidad farmacológica, problemática que se ha empezado a tratar bastante recientemente 
Tabla 6

Resultado de la valoración de los indicadores de adhesión a la guía

\begin{tabular}{|cccccccc|}
\hline & FIABLE & $\begin{array}{c}\text { FÁCIL } \\
\text { OBTENCIÓN }\end{array}$ & PRECISIONN & SENSIBILIDAD & ESPECIFICIDAD & COIILRENCIA & TOTAL \\
\hline 11 & 3 & 3 & 3 & 5 & 4 & 5 & 23 \\
12 & 3 & 3 & 2 & 4 & 4 & 4 & 20 \\
13 & 2 & 3 & 3 & 4 & 3 & 0 & 15 \\
14 & 3 & 3 & 2 & 4 & 5 & 4 & 21 \\
15 & 2 & 1 & 2 & 3 & 0 & 4 & 12 \\
16 & 2 & 1 & 1 & 0 & 4 & 2 & 10 \\
17 & 1 & 1 & 2 & 3 & 3 & 1 & 11 \\
\hline
\end{tabular}

en el ámbito sanitario en general. Con el presente estudio, realizado con los datos de prescripción anuales de un área, se pretende realizar un avance más dentro de la metodología de evaluación de la calidad de prescripción, como parte de un programa global de medición de calidad asistencial en un Sistema Sanitario Público. Para ello, se utiliza como unidad de medida la agregación de datos de facturación de recetas por médicos generales, lo cual es poco habitual en los estudios que reflejan datos de prescripción farmacológica en AP, siendo habitual, sin embargo, la utilización de datos de unidades de mayor tamaño (por ejemplo, equipos de atención primaria) o de áreas sanitarias completas. Se considera más adecuada la utilización de la unidad médico, ya que los resultados son mucho más detallados en cuanto a su contenido, al poder incluirse muchas más variables de relación y se supone un menor margen de error en las conclusiones obtenidas. Se seleccionaron, para nuestro estudio, únicamente médicos generales, debido a que el 96'5\% del gasto farmacéutico del área del año 1996 se debió a prescripciones realizadas por este colectivo, aunque, por supuesto, consideramos que podría haberse hecho estudiando otros facultativos asistenciales dentro del mismo ámbito. Se realizó con datos de un año completo porque durante este periodo la población considerada per- maneció aceptablemente estable en cuanto a contrataciones, incapacidad transitoria, etc. $y$, además, se evitaron las variaciones estacionales, tales como mayor incidencia turística en verano en un equipo que otro o disfrute de vacaciones en distintos periodos.

Obviamente en ningún momento se pretende en este estudio el planteamiento de la idoneidad o no de la selección de medicamentos realizada para el formulario, lo cual sería motivo de un estudio completamente distinto. No obstante, y dado que se ha elaborado mediante un proceso cuidadosamente sistematizado y que se trata de una selección consensuada por un conjunto amplio de profesionales (que eran precisanente los usuarios de la misma), se ha considerado en todo momento que la utilización en un grado elevado de los medicamentos reflejados en dicha selección, sin entrar en su posible indicación, se aproximaría a una calidad de prescripción alta (mayor cficicncia y efectividad).

Los indicadores elaborados para el estudio son sólo ejemplo de unos pocos dentro de una cierta variabilidad posible, teniendo en cuenta los datos disponibles de forma rutinaria y coincidente con los indicadores más empleados en la práctica en este tipo de análisis. Alguno de ellos podría parecer incluso de planteamiento inapropiado desde el inicio, aunque la realidad es que se han utiliza- 
do anteriormente en algún momento y por ello no se han querido desechar. Con esta selección simplemente se pretendía reflejar la complejidad y discrepancia que puede generar la elección de un instrumento destinado a realizar evaluación de calidad. Así, I1, I2 e I4 pretenden - con diferentes matices - reflejar el número relativo de veces que un acto de prescripción se materializaba en un principio activo o especialidad de los previamente seleccionados, aunque lo ideal, presumiblemente, hubiera sido un indicador que utilizara DDD $^{28,29}$, el cual nos es imposible de obtener en estos momentos para el global de la prescripción. El indicador envases de principios activos de la guía/100 personas ajustadas (I3), se elaboró como un indicador paralelo al utilizado en la medida de la prescripción de medicación de baja calidad. El indicador I5 conocemos que se ha seguido en otras áreas y lo hemos encontrado reflejado en algunos trabajos ${ }^{13,14}$, y pretende medir la utilización de un rango estrecho de fármacos mejor conocidos. Los indicadores I6 e 17 no miden el global de los datos de facturación médicos y surgieron para poder valorar el impacto que tuvo la publicación de la guía en la prescripción de los medicamentos de aquellos subgrupos de los que se conoce que hubo más conflicto en la selección, asumiendo que un indicador alto implicaría una mayor intención de adhesión.

De los criterios evaluados, tal vez los más novedosos en cuanto a su aplicación sean los de sensibilidad y especificidad, ya que la medida de indicadores globalizadores en general y de la adhesión a la guía en particular, no proporciona una cualidad binaria, sino que ofrece una gradación cuantitativa, la cual, además, varía en el proceso continuo de medida más o menos rápidamente según las unidades utilizadas. Nos interesa su capacidad, o rapidez, para «alertarnos» cuando se produzca en el tiempo un problema en la misma, o bien que tenga capacidad, rapidez, para identificar un aumento en dicha adhesión (especificidad) a tiempo real, una vez que se han establecido las medidas correctoras. El artificio matemático utilizado también podría estar sujeto a críticas, por falta de exactitud y posibilidad de pequeñas variaciones de los resultados obtenidos, por ejemplo según la estación del año. No obstante, el resultado final buscado (número de meses), permite un margen de error lo suficientemente amplio como para que el método utilizado sea útil. La razón por la que se ha tomado el mes como medida de tiempo necesario para detectar un cambio se debe a que, por las características de obtención de datos de prescripción actuales, nunca podríamos conocer cualquier variación ocurrida en un tiempo inferior. De los resultados obtenidos se deduce que, de hecho, existen diferencias en función de las unidades de medida utilizadas y existen también diferencias en un mismo indicador al considerar separadamente su sensibilidad o su especificidad. Concretamente, en función de si queremos detectar un descenso en la adhesión a la guía -que hemos considerado como un problema de calidad de proceso- o un incremento de la misma, podría proponerse la utilización de distintos indicadores. Se observa también que, por su progresión lineal en el tiempo, la mayoría de los indicadores son más o menos predecibles en su variación, excepto el I5, en el que la misma depende del tiempo de medida acumulado. Esto es lógico si tenemos en cuenta que el número de principios activos (unidad de medida para elaborar I5) es un valor limitado, por lo que en el transcurrir del tiempo cada vez existe menor probabilidad de detectar, y por lo tanto de incorporar a la fórmula, un nuevo principio activo. Se ha evidenciado que la utilidad de 15 para detección de variaciones de la prescripción depende del momento de medida y del volumen de meses acumulados en el indicador, por lo que parece un indicador muy inestable en cuanto a la predictibilidad de la información que ofrece y sólo podría tener utilidad para mediciones puntuales. Sorprende esta observación con la revisión bibliográfica realizada, donde la mayoría de las publicaciones relacionadas con el tema se han basado en una unidad de medida bastante parecida a la de este indicador, sin plantearse su idoneidad. 
La coherencia, que otros autores definen como validez ${ }^{1}$, se ha considerado necesaria de modo concreto para la selección del indicador de adhesión al formulario, aunque pueda no definirse para otros indicadores a los que se pudiera aplicar la metodología de este trabajo. Dicho criterio se planteó como un evaluador de la medida en que los indicadores de seguimiento de la guía proporcionaban información de mejora la eficiencia y/o eficacia de la prescripción, en lugar de dar meramente información del volumen relativo de prescripción coincidente con la selección realizada. Se han considerado variables mixtas gasto, crónicos o compromiso por las siguientes razones: la primera se relaciona tanto con el aspecto económico, como con cl volumen de población atendida, como hemos visto; la segunda puede indicar, por un lado, eficacia en la prescripción (obviamente los medicamentos a los que, según el sistema de financiación vigente, se les ha asignado un $10 \%$ de aportación por el usuario, tienen un aval de su eficacia intrínseca) o bien puede indicar una característica de la población atendida (la cantidad de pacientes con patologías crónicas es una propiedad estructural del cupo de población de cada médico y condicionará el valor de esta variable); la tercera podría implicar, por un lado, una diferencia de actitud en cuanto a esfuerzo de ahorro y de implicación en la calidad del proceso y, por otro lado, es una característica presente durante todo el año del estudio o estructural.

De los resultados obtenidos en el análisis de coherencia, se observa cómo alguno de los indicadores (I3) que se habían planteado en principio como posible instrumento de medida, se descalifican por sí solos, tanto en las correlaciones simples como en la regresión multivariante, al detectarse claramente que no está relacionado positivamente con las variables indicadoras de eficiencia y/o eficacia [B1], sino todo lo contrario, lo cual nos confirma una vez más la necesidad de realizar un estudio detallado de la información proporcionada por los instrumentos utilizados en la evaluación cualitativa de cualquier proceso. También se observa cómo otros indicadores, claramente relacionados con las variables de B1 y que pudieran en principio parecer casi equivalentes, sin embargo, muestran diferencias a la hora del análisis multivariante, que pueden ser determinantes de su elección. Como curiosidad, incluso, encontramos relaciones que nos pueden dar idea de la naturaleza de la medida realizada, como la correlación negativa en el análisis bivariante del indicador I5 con las variables de población, que parece orientarnos de un modo lógico, por otra parte, hacia el hecho de que un factor influyente en la diversificación de la prescripción es el volumen de población atendida. Otros resultados, como la importante implicación de algunas variables estructurales en la explicación de la variabilidad de ciertos indicadores, no se han sabido valorar $y$, en todo caso, influyen muy poco para los resultados que se pretenden evaluar. También destaca entre los resultados obtenidos en el análisis de la coherencia, la clara correlación negativa entre los indicadores que incluyen datos globales de prescripción con cl gasto farmacéutico por persona, relación similar a la que ya se había obtenido previamente en la literatura anglosajona ${ }^{7,27,30,31}$. A partir de este resultado se podría intuir la posibilidad de racionalizar el gasto farmacéutico mediante la claboración consensuada de formularios locales, sin que ello suponga una restricción de las posibilidades de trabajo de los prescriptores ni una merma en la satisfacción por la asistencia sanitaria recibida por los usuarios aunque, para la ejecución de esta estrategia, parece necesario reforzar la información impartida tanto a profesionales sanitarios como a público en general ${ }^{31-33}$.

Otros criterios a medir presentes en la bibliografía, como la relevancia o la justificación científica ${ }^{19}$ no se han incluido en nuestro análisis, porque se ha considerado que no proporcionan diferencias entre distintos indicadores aplicados a un mismo criterio de evaluación, pero han de tenerse en cuenta al valorar una batería de indicadores aplicados a distintos criterios. 
Con este estudio se ha demostrado que la evaluación de la calidad de la prescripción se debe realizar de un modo crítico y tras un periodo de reflexión y análisis detallado de los objetivos que perseguimos y de los instrumentos que vamos a utilizar, ya que van a ser condicionantes de los métodos y técnicas más oportunas para implementar dicha medición. El método propuesto se basa en una evaluación lo más objetiva posible de las características relevantes exigidas al indicador a seleccionar, evitando así selecciones basadas sólo en la intuición. Dicho método se propone como un intento de sistematizar la selección de cualquier tipo de indicador que utilice mediciones continuas globales o parciales de datos de prescripción o facturación de medicamentos, con el objeto de facilitar el planteamiento de soluciones dinámicas $u$ otros mecanismos para profundizar en el conocimiento del posible problema. Su aplicación al caso concreto de la medición de la adhesión a la guía ha proporcionado la propuesta del indicador del porcentaje de recetas de principios activos de la guía con respecto al total de recetas, como más apropiado para efectuar dicho seguimiento frente al resto de los indicadores evaluados. Con el mismo proceso, se ha comprobado cómo, efectivamente, la guía parece un instrumento capaz de ser un apoyo hacia una mayor eficiencia en la farmacoterapia.

\section{AGRADECIMIENTOS}

Jose Ramón Saldaña Alonso, informático del área y autor de varios programas utilizados para la elaboración de este estudio; María José Gómez Castro y Juan Rubio Domínguez, médicos del Área de Avilés y compañeros de trabajo que han revisado, corregido y aportado sugerencias para el presente trabajo; Miguel Siles Gutierrez, inspector farmacéutico de la Dirección Territorial de Zaragoza y autor de la codificación y asignación de DDD de principios activos que se han utilizado.

\section{BIBLIOGRAFÍA}

1. Agra Varela Y, García Boró S. Evaluación y mejora de la calidad asistencial (V) Estudios de monitorización. Medifam 1998; 8: 390-8.

2. Saturno Hernández PJ. Monitorización del uso de medicamentos para mejorar la calidad de prescripción. Problemas, métodos e indicadores. Aten Primaria 1996; 18: 331-338.

3. Saturno Hernández PJ, Gascón Cánovas JJ. Validez de la utilidad terapéutica y el valor intrínseco como indicadores de la calidad de la prescripción farmacéutica: análisis de los tratamientos en casos de resfriado común. Aten Primaria 1997; 19: 400-6.

4. Amado Guirado E, Madrilejos Mora R, Pérez Rodríguez MT, Catalán Ramos A. Análisis cualitativo de la prescripción: una propuesta de indicadores por niveles. Comunicación oral al I Congreso de la SEFAP; 1996, octubre.

5. Amado A, Madrilejos R, Pérez MT, Catalán A. Análisis de la prescripción en Atención Primaria: evaluación de un sistema de indicadores. Aten Primaria 1997; 20:381-384.

6. Gomez Juanes V y col. Programa de mejora de la prescripción farmacológica en atención primaria. Madrid : Insalud; 1998 .

7. Hill Smith I. Sharing resources to create a district drug formulary: a countywide controlled trial. Br J Gen Prac 1996; 46: 271-5.

8. Azagra Ledesma $R$ y col. Seguimiento en la calidad de la prescripción de medicamentos: el CAP de Ciudad Badía (1986-1990). Aten Primaria 1992; 10: 707-710.

9. Avery AJ, Walker B, Heron T, Teasdale SJ.. Do prescribing formularies help GPs prescribe from a narrower range of drugs? A controlled trial of the introduction of prescribing formularies for NAIDs. Br J of Gen Prac 1997; 47: 810-814.

10. Stewart D, Krska J, Downie G. Adherence to the Grampian joint drug formulary in general practice. J Clin Pharm Ther 1996; 21:79-82.

11. Field J. How do doctors an patients react to the introduction of a practice formulary?. Fam Prac $1989 ; 6: 135-40$

12. Kochen MM, Sandoholzer H, Himmel W. Attitudes of primary acare physicians towards the use of a drug formulary - preliminary results of 
a study in Germany. Int J Clin Pharmacol Ther 1994; 32: 400-402.

13. Guía Farmacoterapéutica de Atención Primaria Área III de Salud, Avilés-Asturias. Oviedo: Insalud; 1996.

14. Grant GB, Gregory DA; van Zwanenberg TD. Development of a limited formulary for general practice. Lancet 1985; 1(8436):1030-2.

15. Catalán Ramos A. Metodología para la elaboración de formularios de medicamentos en la Atención Primaria. Aten Primaria 1993; 12: 227-231.

16. Comisión de Uso Racional del Medicamento del Área III de A.P. - Avilés, Asturias. Protocolo para la elaboración de la guía farmacológica de Atención Primaria del Area III - Avilés; 1993.

17. Janknegt R. Formulary choice of fluorquinolones. Use of clinical data in objectified judgement analysis: results from 33 countries. EHP 1996; 2:17-20.

18. Janknegt $R$ et al. HMG- coenzyme A reductasa inhibitors: drug selection by means of the SOJA method. EHP 1999; 5: 29-43.

19. Gómez Juanes V y col. Indicadores de calidad en la prestación farmacológica. Madrid: Insalud; 1994.

20. WHO Collaborating Centre for Drugs Statistics Methodology. ATC Index with DDD's 1997. Oslo: WHO; 1997.

21. Torralba M, Calero MI, González JA, López M. Nivel cultural y envejecimiento de la población, formación y dependencia del médico, y tipo de centro como condicionantes de la prescripción. Farm Clin 1995; 12: 378-86.

22. Catalán A, Parellada N,. Variables que intervienen en la despesa farmacèutica dels equips d' Atenció Primària. Anàlisi y possibilitats de gestió. Barcelona: Generalitat de Catalunya; 1995.

23. Catalán Ramos A, Madrilejos Mora R, Font i Pous M, Pané Mena $O$, Jiménez Villa J, Huguet Recasens M. Factores asociados a la prescripción de medicamentos. Gac Sanit 1989; 3: 497501.
24. Jiménez Puente A, Ordóñez Martí-Aguilar MV, Córdoba Doña JA, Fernández Gómez MA. Factores relacionados con el gasto y la calidad de prescripción farmacéutica en Atención Primaria. Aten Primaria 1995; 16: 131-136.

25. Solano V M, Dolsac I, Lapeña R, Arnal J M. Análisis de las variables que determinan el gasto farmacéutico de Atención Primaria. Comunicación a las XVIII Jornadas de Economía de la Salud; mayo 1998; Vitoria.

26. Morton - Jones TJ. Pringle MAL. Prescribing costs in dispensing practices. BMJ 1993; 306: 1244-6.

27. Majeed A, Cook D, Evans N. Variations in general practice prescribing costs - implications for setting and monitoring prescribing budgets. Health Trends 1996; 28: 52-5.

28. Gómez Juanes V, Benedí González A, Crespo Sánchez-Eznarriaga B. Análisis de medicamentos en el Sistema Nacional de Salud, utilizando la DDD como unidad de medida. Comunicación al II Congreso de la Sociedad Española de Farmacéuticos de Atención Primaria; octubre 1997; San Sebastián.

29. Maxwell M, Heaney D, Howie JGR, Noble S. General practice fundholding: observations on prescribing patterns and costs using teh defined daily dose method. BMJ 1993; 307: 1190-4.

30. Beardon PHG, Brown SV, Mowat DAE, Grant $\mathrm{JA}, \mathrm{McDevitt}$ DG. Introducing a drug formulary to general practice effects on practice prescribing costs. J R Coll Gen Pract (Occas Pap) 1987; 37:305-7.

31. Dowell JS, Snadden D, Dunbar JD. Changing to generic formulary: how one fundholding practice reduced prescribing costs. BMJ 1995; 310:505-8.

32. Black J, Griffin T, Beisel W, Bartels MD. Implementation of an outpatient prescription drug formulary in a managed-care system. Am J Hosp Pharm 1988; 45: 561-5.

33. Rucker TD, Schiff G. Drug formularies: mythsin-formation. Med Care 1990; 28(10):928-42. 